\title{
The Human Microbiota and Its Relationship with Allergies
}

\section{Fyhrquist, Nanna}

2019-09

Fyhrquist, N 2019 , ' The Human Microbiota and Its Relationship with Allergies ' ,

Gastroenterology Clinics of North America , vol. 48 , no. 3 , pp. 377-+ . https://doi.org/10.1016/j.gtc.2019.04.005

http://hdl.handle.net/10138/319798

https://doi.org/10.1016/j.gtc.2019.04.005

unspecified

publishedVersion

Downloaded from Helda, University of Helsinki institutional repository.

This is an electronic reprint of the original article.

This reprint may differ from the original in pagination and typographic detail.

Please cite the original version. 


\section{The Human Microbiota and Its Relationship with Allergies}

Nanna Fyhrquist, $\mathrm{PhD}^{\mathrm{a}, \mathrm{b}, *}$

\section{KEYWORDS}

- Allergic disease $\bullet$ Human microbiota $\bullet$ Dysbiosis $\bullet$ Biodiversity hypothesis

\section{KEY POINTS}

- Perturbations in the human microbiome may have profound effects on the host, which may result in developing chronic inflammation and disease, especially in genetically predisposed individuals.

- The mechanisms via which the human microbiota may be regulating the immune system, either by providing protective signals or stimulating effector mechanisms, are only beginning to be understood.

- Many allergic conditions are associated with dysbiosis; however, it remains unclear whether it is the cause or an epiphenomenon of the disease.

\section{INTRODUCTION}

Trillions of microorganisms thrive on and inside bodies and have done so, participating in the shaping of physiology through millions of years of common evolution. The relationship between humans and their microbiota is truly mutualistic, with microorganisms playing a significant role in host digestion, metabolism, and the immune system. Several lines of evidence indicate that the human-associated microbes have been selected based on coadaptation. ${ }^{1}$ Initially, microorganisms were regarded as mere pathogens, which cause infections and disease and which the host immune system should recognize and eliminate. It has become increasingly clear, however, that microorganisms influence most aspects of human physiology, and, conversely, the host immune system has largely developed to support the mutualistic relationship between the host and these highly diverse microbes. This interaction is extensive, as

Disclosure Statement: Dr. N. Fyhrquist received funding support from Svenska kulturfonden, the Jane and Aatos Erkko Foundation and the FP7/2007-2013 grant 261366.

a Institute of Environmental Medicine, Karolinska Institutet, Box 210, Stockholm 17177, Sweden; ${ }^{b}$ Department of Bacteriology and Immunology, Medicum, University of Helsinki, Helsinki, Finland

* IMM, Box 210, Stockholm 17177, Sweden.

E-mail address: nanna.fyhrquist@ki.se 
evidenced by a study comparing germ-free mice with conventionally raised mice, revealing that most metabolites in host blood are derived from the gut microbiota. ${ }^{2}$ Therefore, significant perturbations in the microbial community, or dysbioses, may have profound effects on the host, which may result in developing chronic inflammation and disease, especially in genetically predisposed individuals.

The composition of the human microbiota is largely determined by anatomic site, with some interpersonal variation. Thus, individuals can be grouped according to the occurrence of major phyla at specific body sites. Moreover, there is temporal variability in the composition, influenced by diet, season, or changes in the state of physiology, including regularly occurring fluctuations, such as the menses, or isolated phenomena, such as pregnancy. ${ }^{3,4}$ Nevertheless, the microbiota of 1 individual usually varies fairly narrowly, except when significantly perturbed by agents, such as antibiotic treatments or infections. Such episodes can lead to a new transient equilibrium or even a new stable state. The concept of resilience, that is, the capacity of an ecosystem to recover from perturbations, is central in the context of health and the human microbiota. Although adult microbiotas usually are highly resilient, children are more vulnerable, and frequent perturbations may lead to increasingly impaired recovery each time, with implications for health.

Chronic inflammatory diseases are on the rise worldwide today; the central role of the immune system in driving disease is only beginning to be understood. Yet, how the environment, including the human microbiota, may trigger this development, is unknown. For many conditions and illnesses, a challenge is to discover whether there is a causal link between variations in the microbiota and pathology. It has been hypothesized that although human ecology changes, so does the microbiota, with implications for health. A modern lifestyle, including large-scale urban living, cesarean sections, replacement of breast milk with formula, cleaner drinking water, smaller families, and frequent antibiotics, has brought with it dramatic changes to the transmission and maintenance of the indigenous microbiota, resulting in long-term effects on its composition and stability. And if the microbiota affects physiology, and its composition has changed, it should be no surprise that this might alter host homeostasis and, as a consequence, disease risk. ${ }^{5}$

\section{ALLERGIES AND IMMUNOREGULATION BY THE HUMAN RESIDENTIAL MICROBIOTA}

Allergic diseases, including asthma, rhinitis, atopic dermatitis (AD), and food allergies, have been increasing to epidemic proportions during the past century, especially in high-income countries. The idea that there might be a link between the allergy epidemic and reduced microbial exposure was first proposed in 1989. The socalled hygiene hypothesis, formulated by Dr David Strachan, ${ }^{6}$ suggested that a lower frequency of infections in early childhood could explain the rise in atopic diseases during the twentieth century. Later on, the hygiene hypothesis was refined to include the old friends hypothesis, ${ }^{7}$ which suggests that it is not the infections that protect against allergies; rather, the allergy epidemic is a result of a loss of sufficient interaction with health-promoting microbes-old friends-which have been present through thousands of years of common evolution and the evolvement of the mammalian immune system. Moreover, the hygiene hypothesis was expanded in 2010 by von Hertzen and colleagues ${ }^{8}$ into the biodiversity hypothesis, which suggests that, in addition to microbes in the home, in food, in drinking water, and on domestic animals, microbes of the living environment, in general, may play a key role in shaping the composition of microbial communities on the skin, in the respiratory system, and in the gut, with consequences for physiology and health. 
Old friends include usually nonharmful commensals, with which humans coevolved, because they performed crucial physiologic functions. Moreover, humans evolved to tolerate other harmless organisms, which inevitably were taken daily into the body in large quantities, and also organisms that induced low-level infections but were tolerated, such as helminths, Salmonella, hepatitis A virus, and Helicobacter. pylori. Some of these organisms, such as blood nematodes, can be harmful, but, once established, trying to eliminate them may cause pointless immunopathology. These microbes continuously stimulate the immune system via microbe-associated molecular patterns or pathogen-associated molecular patterns and cognate pattern recognition receptors, and it is natural to conceive that the immune system evolved to detect and eliminate these potentially dangerous organisms. What is less obvious, however, is that microbial exposures at the same time also serve to down-regulate the immune system, preventing it from developing inappropriate inflammatory responses against self, harmless allergens, and gut contents. These are all targets in autoimmune diseases, allergic disorders, and inflammatory bowel diseases. Moreover, the regulatory mechanisms should be able to shut down ongoing inflammatory responses and downregulate unnecessary background inflammation. ${ }^{9}$

The mechanisms via which the human residential microbiota may be regulating the immune system are only beginning to be understood. These include secreted molecules or metabolites, such as short-chain fatty acids (SCFAs), which can induce the differentiation of regulatory dendritic cells (DCs) or the expansion of regulatory T-cell (Treg) populations. ${ }^{10,11}$ Moreover, microbes can influence the immune system through the induction of helper T cell type $1\left(T_{H}\right)$-type immune responses, which inhibit the development of $T_{H} 2$ cells and thereby protect against helper T cell type $2\left(T_{H} 2\right)-$ driven allergies. Endotoxins stimulate macrophages and antigen-presenting cells to produce interleukin (IL)-12, which triggers the development of $T_{H} 1$ immunity. Microbe-induced programming might further involve epigenetic modifications at immune-related genes, including histone acetylation. ${ }^{12}$

\section{THE GUT MICROBIOTA AND THE DEVELOPMENT OF ATOPIC DISEASE}

The largest collection of human bacteria, by far, is found in the distal gut, consisting of 500 to 1000 different species, including the phyla Bacteroidetes, Firmicutes, Actinobacteria, Proteobacteria, and Verrucomicrobia. The host immune system has evolved to cope with the massive microbial presence by learning to judge correctly between rejecting or accepting new species and to optimally control retained species. In turn, the gut microbiota has developed strategies to both reinforce resistance against harmful invaders and to avoid rejection by the host. These include boosting of host defense as well as the induction of regulatory mechanisms, including the activation of Tregs. Treg induction seems to be a widespread feature of microbiota colonization. ${ }^{13}$ The bacterium Bacteroides fragilis, for instance, is able to drive the differentiation of IL10-secreting Tregs, by producing an unusual capsular polysaccharide $A,{ }^{10}$ and members of Clostridia metabolize SCFAs, which can have systemic antiinflammatory effects, through influencing DCs and T-cell responses. Segmented filamentous bacteria (SFB) is an example of the opposite, an organism that is able to promote effector responses through driving $\mathrm{T}_{\mathrm{H}} 1$ and $\mathrm{T}_{\mathrm{H}} 17$ immunity. ${ }^{14}$ SFB has a specific feature of adhering strongly to the epithelium of the ileum and Peyer patches, ${ }^{15}$ in contrast to most other members of the microbiota, which remain entrapped in the mucus and have little or no physical contact with host epithelium. This feature facilitates sampling and presentation of SFB antigens to T cells by DCs and likely explains the unusual ability of SFB to influence host immunity. Although SFB-deficient mice 
lack $T_{H}$ 17-type responses and are susceptible to bacterial infections, it is evident that SFB plays a key role in strengthening the gut barrier.

Several studies underline the importance of the composition of the gut microbiota in early life for proper gut development, immune cell maturation, and resistance to pathogens. ${ }^{16}$ Moreover, early-life dysbiosis of the gut microbiota has been linked with an increased risk of chronic inflammatory disorders, including allergies, later in life. The mode of delivery is important, with babies born by cesarean section colonized by more Staphylococcus, Corynebacterium, and Propionibacterium species and fewer Bacteroidetes and Actinobacteria compared with vaginally delivered babies, who are enriched for Clostridia. ${ }^{17}$ Cesarean birth is linked to predisposition toward asthma during childhood, ${ }^{18}$ and studies show that children with an altered or less diverse gut microbiome composition early in life are more likely to develop asthma. ${ }^{19-21}$ Moreover, perturbations of the gut microbiome, such as colonization by Clostridium difficile at an early age, is significantly associated with the development of wheeze, and recurrent antibiotics, having an impact on on the diversity of the microbiota early in life, ${ }^{22}$ correlate with the development of asthma. ${ }^{23}$ In contrast, breastfeeding is inversely related with the risk of developing of asthma, ${ }^{24}$ and exposure to environmental microbes or farming environments has been associated with protection against asthma. ${ }^{25-27}$ It remains to be seen, however, whether the latter also is linked to shifts in communities in the intestines, and whether the gut microbiota can directly drive atopic disease or confer protection against disease remains unclear.

\section{THE CUTANEOUS MICROBIOME AND ATOPIC ECZEMA}

The skin is colonized by trillions of microorganisms at a density of $1 \times 10^{6}$ microbes per square millimeter. The composition of the skin microbiota is dependent on the physiology of the site, with sebaceous sites dominated by lipophilic organisms, such as Propionibacterium species, and humid environments inhabited mainly by Staphylococcus and Corynebacterium species. ${ }^{28}$ Skin communities are stable over time, especially in the more sheltered areas, despite external challenges, such as sudden changes in temperature, windiness, humidity, clothing, sweating, or the use of skin products. Studies have shown that this stability is dependent on the maintenance of species and strains over time rather than on the acquisition of species from the environment. ${ }^{29}$ Compared with the gut, the skin is a hostile environment to microbes - scarce in nutrients, high in salt concentration and antimicrobial peptides, and endowed with a low $\mathrm{pH} .{ }^{30}$ Nevertheless, a few phyla have adapted well to these conditions, including Actinobacteria, Bacteroidetes, Firmicutes and Proteobacteria - which also are abundantly present in the gut, but at different proportions compared with the skin.

Similar to the gut microbiota, the skin microbiota is essential in the protection against invaders and in educating the skin immune system as well as in skin metabolism and xenometabolism. ${ }^{31}$ Skin microbes contribute to the maintenance of a physical and immune barrier of the host and are able to induce the production of antimicrobial peptides and activate complement, and regulate the levels of IL- $\beta$ produced by keratinocytes and local antigen-presenting cells (APCs), which in turn control the capacity of resident T cells to produce IL-17 and interferon- $\gamma .^{32}$ Thus, analogous to the gut microbiota, skin microorganisms can act as adjuvants to the skin immune system.

One of the functions of normal skin microbiota is to suppress the growth of the pathogen Staphylococcus aureus, utilizing several mechanisms. For instance, S epidermidis can generate peptide antibiotics (lantibiotics) with bactericidal effects against $S$ aureus. Moreover, a lantibiotic produced by $S$ hominis, can act synergistically with 
host antimicrobial peptides. S epidermidis also is able to release phenol-soluble modulins, which specifically inhibit $S$ aureus and act cooperatively with host antimicrobial peptides. Moreover, Propionibacterium acnes may inhibit the growth of $S$ aureus, through the generation of fatty acids by fermenting glycerol in sebum, which lowers the local $\mathrm{pH}$ in skin. ${ }^{33}$ These bacteria are common on healthy skin but typically lacking in inflamed skin, especially in AD.

Whether the microbiota is able to induce antiinflammatory responses in the skin remains unclear. Skin contains the highest frequencies of Tregs in the body, and a large fraction of these can be found in the vicinity of hair follicles. ${ }^{34}$ In neonates, but not in adults, the colonization by $S$ epidermidis results in an influx of activated Tregs, and blocking their entry prevents tolerance to commensals. ${ }^{35}$ Tregs play an important role in calibrating the immune response to microorganisms, but, although the skin microbiota continuously undergoes shifts defined by developmental stages and in the context of disease and infections, it remains unknown how the responses to these microbial fluctuations are controlled. There are some examples of skin commensals that promote regulatory responses, including Vitreoscilla filiformis, originally found in thermal spa water, which can promote the accumulation of Tregs in the skin and protect against $\mathrm{AD} .{ }^{36}$ Moreover, S epidermidis is known to induce IL-10 production by DC in vitro, ${ }^{37}$ and Acinetobacter /woffii is able to promote both IL-10 production and $\mathrm{T}_{\mathrm{H}} 1$ responses in the skin, conferring systemic protection against allergic sensitization and inflammation in a mouse model. ${ }^{38}$

$A D$ is a chronic inflammatory skin disease, characterized by intense itch and recurrent, inflammatory eczematous lesions. ${ }^{39} A D$ is one of the most common chronic diseases and affects up to one-fifth of the population in developed countries. Previously, $A D$ pathogenesis was believed to be mediated primarily by abnormalities of humoral and T-cell-mediated immunity, but recently, the epidermis and its barrier functions have been placed at the forefront of research and management efforts. The strongest risk factor of $A D$ is a positive family history for atopic diseases, ${ }^{40}$ and systematic gene mapping studies have identified multiple risk loci for $A D$. For instance, mutations in the filaggrin gene (FLG) gene, which is involved in the formation of the epidermal skin barrier, have been identified as major predisposing factors for atopic eczema. ${ }^{41}$ The, to date, approximately 30 identified susceptibility loci, however, explain less than $20 \%$ of the estimated heritability. For instance, most patients with AD do not have any FLG mutations, and up to $60 \%$ of FLG mutation carriers do not develop atopic disease. Many of the risk genes contribute to immune abnormalities, in particular innate immune signaling and T-cell activation. Most of them also are linked, however, to other inflammatory diseases, suggesting that atopy nonspecific processes are involved. Instead, environmental factors, such as the living environment, lifestyle, and diet, are believed to drive the inherited disease susceptibility predisposition into manifestation. Although the incidence of $A D$ has increased by 2 -fold to 3 -fold during the past decades in industrialized countries, ${ }^{42}$ it has been suggested that a westernized lifestyle may influence the disease outcome. Few proposed environmental risks are supported, however, by strong epidemiologic data, and, in general, little is known about how inherited and environmental factors interact and how this pertains to disease pathophysiology, course, and outcomes.

The skin microbiota frequently is disrupted in $A D$, with the diversity of the microbiota greatly reduced in favor of the genus Staphylococcus $-S$ aureus, in particular. AD is a chronic, relapsing condition, with shifts in the microbiota associated with disease flares. ${ }^{43} S$ aureus is an established trigger to disease,${ }^{44}$ but how the skin microbiota modulates disease is poorly understood. Treatment with antibiotics often is successful, with decreasing disease severity along with the reduction of $S$ aureus 
abundance. ${ }^{45} S$ aureus is known to express several molecules, which may contribute to the intensity of the symptoms, including delta toxin, which stimulates mast cells, and alpha toxin, which damages the skin barrier through destroying keratinocytes. $S$ aureus also is able to generate phenol-soluble modulins, which stimulate cytokine release; proteases, which damage the epidermal barrier; and superantigens, which give rise to specific $\operatorname{lgE}$ and exacerbate the inflammatory response through nonspecific activation of $\mathrm{T}$ cells. ${ }^{46,47}$ Recent studies reveal specific differences between staphylococcal strains in the capacity to elicit skin inflammation, and certain strains are particularly able and better suited than others at colonizing the inflammatory skin site in $A D$ skin, ${ }^{48,49}$ which contributes to the complexity of AD.

It remains unknown which host or microbiome-derived mechanisms either promote or inhibit the colonization of $A D$ skin by $S$ aureus and what the conditions are that trigger changes to the skin microbiota at the beginning of an AD flare. S. aureus usually dominates the bacterial skin community in $A D$, along with an extensive loss of other potentially beneficial species of bacteria. These bacteria may contribute to a healthy skin environment through the generation of short chain fatty acids, which may function both as relevant natural moisturizing factors and through lowering the skin $\mathrm{pH} .{ }^{33}$ Moreover, certain gram positive anaerobic cocci (GPACs), including Finegoldia, stimulate rapid induction of antimicrobial peptides in human keratinocytes, constituting an important signalling mechanism to the keratinocytes when the skin is injured. ${ }^{50}$ Therefore, the complete or partial absence of these potentially beneficial microbes may exacerbate a dry and alkaline skin surface environment, and may cause impaired danger signalling in the skin, priming it for further pathogen growth and inflammation.

\section{THE RESPIRATORY MICROBIOME AND ASTHMA}

Until recently, the lung has been considered a sterile organ lacking microbial inhabitants. Now, however, it has become evident that the healthy respiratory mucosa is colonized by a range of bacterial communities. ${ }^{51}$ The relative abundance and load of these phyla differ significantly from that of other body compartments, with the lower respiratory tract among the least populated surfaces of the human body, with an estimated number of 10 to 100 bacteria per 1000 human cells. The 2 predominant phyla in the airways are Firmicutes and Bacteroidetes, with Actinobacteria, Proteobacteria, and Fusobacteria minor constituents of the local microbiota. Like elsewhere on mucosal surfaces, a balance between immune tolerance and inflammation must be maintained in the lung and is in part regulated by the cross-talk between immune cells and the microbiome. ${ }^{52}$ Changes in the microbial composition may negatively influence the immune homeostatic networks, which has been clearly demonstrated in germ-free mice, which display impaired tolerance and increased susceptibility to inflammation or infections. ${ }^{53}$ Likely there is substantial cross-talk between mucosal sites, in particular the lung and the gut, because chronic lung disorders often exhibit intestinal disease manifestation, and, conversely, respiratory infections often are accompanied by intestinal indications. ${ }^{54}$

The composition of the airway microbiota develops extensively from the time point of birth and is influenced by the environment. Although it is clear that the microbiome significantly influences host immune maturation and activity, it remains unclear to what extent patterns of airway microbial dysbiosis actually drives disease. Birth mode (vaginal or cesarean section) greatly influences the development of the gut, skin, and respiratory microbiota, with implications for health later on, and the exposure to environments conferred with a high microbial burden and diversity have proved to influence the risk of developing atopic disease and asthma. ${ }^{26,55}$ 
Mouse models clearly show that the microbiome has a role in the development of airway diseases. One study has shown that the susceptibility to house dust mite (HDM)-induced lung inflammation in a mouse model is dependent on age and associated with the gradually increasing load of Bacteroidetes in the lung. The younger mice that were exposed to HDM generated significantly more exaggerated $\mathrm{T}_{\mathrm{H}} 2$-type responses compared with older mice. ${ }^{56}$ The protective effect in the older mice was associated with an increased number of bacteria in the airways and a shift from predominance of Gammaproteobacteria and Firmicutes to Bacteroidetes. Oral supplementation of beneficial microbes, such as Bifidobacterium, or microbial metabolites, such as SCFAs, similarly has provided protection against allergic sensitization and inflammation, providing evidence that the gut and lung are closely linked in this context. Several studies also have suggested that direct exposure of the respiratory epithelium to microbial products provides some protective effects. For instance, exposure to dust from farm environments protects mice against HDM-induced asthma via A20-dependent mechanisms in the airway epithelium. ${ }^{55}$ Furthermore, Stein and colleagues ${ }^{26}$ have shown that exposure of mice via the airways to dust from Amish houses, but not Hutterite houses, protect against asthma in mice, via MyD88-dependent and Trif-dependent mechanisms.

In humans, the risk of asthma is associated with lifestyle and microbial exposures, and early-life dysbiosis of the gut microbiota has been consistently associated with the increased risk of asthma later in life. Asthma recently has been shown to associate also with a significant dysbiosis in the airway microbiota, including an increase in the diversity the phylum Proteobacteria, ${ }^{57}$ with the appearance of families, such as Comamonadaceae, Sphingomonadaceae, and Pseudomonadaceae. These results are still controversial, however, and further clinical trials and microbiome studies are needed.

\section{THE KARELIA STUDY}

In the author's study, focusing on children in Finnish and Russian Karelia, allergic symptoms and atopic diseases are systematically more common in Finnish children and adults compared with their Russian counterparts. These 2 adjacent areas are geologically and climatically similar but socioeconomically highly distinct. In Russian Karelia, hay fever is almost nonexistent, and only $2 \%$ of the children are sensitized to birch pollen. In Finland, approximately one-sixth of the children suffer from hay fever, and $27 \%$ are sensitized to birch pollen. ${ }^{58}$ On the Finnish side, the author showed that biodiversity of the living environment is related to the composition of the microbiota on the skin in these children and the risk of developing allergic disease. Furthermore, the author observed that the abundance on the skin of the phylum Gammaproteobacteria down to the genus-level Acinetobacter in these children was significantly associated with the capacity of the immune cells in peripheral blood to produce antiinflammatory cytokine $\mathrm{IL}-10,{ }^{25}$ feeding into a network of regulatory gene expression. The author further examined the capacity of Acinetobacter to modify immune responses in vitro and in vivo and found that compared with other human commensals, including $S$ epidermidis and E. coli, Acinetobacter species were particularly potent at inducing antiinflammatory and $T_{H} 1$-type immune responses in human keratinocytes and human monocyte-derived DCs as well as locally in the skin in a mouse model, protecting against systemic allergic sensitization and inflammation. ${ }^{38}$ Both the diversity and the abundance of Acinetobacter species on the skin and on the nasal epithelium were significantly higher in Russian children compared with children in Finnish Karelia, linking cutaneous Acinetobacter with the unusually low prevalence of allergic disease in the Russian area. ${ }^{59}$ 
Finally, to establish causality between exposure to environmental biodiversity and the tuning of host microbiomes and the immune system, the author exposed mice to soil for 2.5 months, using siblings kept on clean bedding as controls. Exposure to soil modified the gut microbiota of the mice extensively, including a significant shift in its composition including an increased abundance of Bacteroidetes relative to Firmicutes. A low ratio of Bacteroidetes to Firmicutes in the gut microbiota previously was linked by several studies to various inflammatory conditions, such as obesity ${ }^{60}$ and diabetes ${ }^{61}$ and the development of asthma in human babies. ${ }^{62,63}$ Moreover, in the soil exposed mice, the author observed significantly up-regulated expression of antiinflammatory signaling in the intestinal epithelium of the ileum, which is the most distal part of the small intestines. The ileum is an immunologically highly active tissue, containing numerous Peyer patches, which are engaged in the surveillance of microorganisms in the intestinal lumen and facilitate the generation of immune responses within the mucosa. ${ }^{64}$ When exposing the mice to the experimental asthma protocol, the author observed that the inflammatory response was significantly alleviated in the soil exposed mice. ${ }^{27}$ Thus, the author's work provides evidence of the role of environmentally acquired microbes in modifying the gut microbiota, and alleviating $\mathrm{T}_{\mathrm{H}} 2$-driven allergic inflammation.

\section{SUMMARY}

The living environment has profound influence on human health, mediated in part by modifications in the human microbiota. The human microbiota in turn influences both the development and the calibration of the immune system, with consequences for human health. The prevalence of allergies has reached epidemic levels during the past 4 decades, especially in high-income countries, and there is evidence that this phenomenon might be linked to reduced exposures to beneficial environmental microorganisms, and allergic disease often is associated with perturbations in the human microbiota. It remains unclear, however, if microbial dysbiosis actually can drive relevant disease mechanisms or if it simply reflects associated phenomena, such as altered patterns of immune responses to microbes and environmental stimuli.

\section{REFERENCES}

1. Ochman $\mathrm{H}$, Worobey $\mathrm{M}, \mathrm{Kuo} \mathrm{CH}$, et al. Evolutionary relationships of wild hominids recapitulated by gut microbial communities. PLoS Biol 2010;8:e1000546.

2. Wikoff WR, Anfora AT, Liu J, et al. Metabolomics analysis reveals large effects of gut microflora on mammalian blood metabolites. Proc Natl Acad Sci U S A 2009; 106:3698-703.

3. Bogaert D, Keijser B, Huse S, et al. Variability and diversity of nasopharyngeal microbiota in children: a metagenomic analysis. PLoS One 2011;6:e17035.

4. Ravel J, Gajer P, Abdo Z, et al. Vaginal microbiome of reproductive-age women. Proc Natl Acad Sci U S A 2011;108(Suppl 1):4680-7.

5. Blaser MJ. Who are we? Indigenous microbes and the ecology of human diseases. EMBO Rep 2006;7:956-60.

6. Strachan DP. Hay fever, hygiene, and household size. BMJ 1989;299:1259-60.

7. Rook GA. 99th Dahlem conference on infection, inflammation and chronic inflammatory disorders: darwinian medicine and the 'hygiene' or 'old friends' hypothesis. Clin Exp Immunol 2010;160:70-9.

8. von Hertzen L, Hanski I, Haahtela T. Natural immunity. Biodiversity loss and inflammatory diseases are two global megatrends that might be related. EMBO Rep 2011;12:1089-93. 
9. Rook GA, Raison CL, Lowry CA. Microbial "Old Friends", immunoregulation and socio-economic status. Clin Exp Immunol 2014;177(1):1-12.

10. Round JL, Mazmanian SK. Inducible Foxp3+ regulatory T-cell development by a commensal bacterium of the intestinal microbiota. Proc Natl Acad Sci U S A 2010; 107:12204-9.

11. Atarashi K, Tanoue T, Oshima K, et al. Treg induction by a rationally selected mixture of Clostridia strains from the human microbiota. Nature 2013;500:232-6.

12. Brand $\mathrm{S}$, Teich $\mathrm{R}$, Dicke $\mathrm{T}$, et al. Epigenetic regulation in murine offspring as a novel mechanism for transmaternal asthma protection induced by microbes. J Allergy Clin Immunol 2011;128:618-25.e1-7.

13. Geuking MB, Cahenzli J, Lawson MA, et al. Intestinal bacterial colonization induces mutualistic regulatory T cell responses. Immunity 2011;34:794-806.

14. Ivanov II, Atarashi K, Manel N, et al. Induction of intestinal Th17 cells by segmented filamentous bacteria. Cell 2009;139:485-98.

15. Klaasen HL, Koopman JP, Van den Brink ME, et al. Intestinal, segmented, filamentous bacteria in a wide range of vertebrate species. Lab Anim 1993;27: $141-50$.

16. Sommer F, Backhed F. The gut microbiota-masters of host development and physiology. Nat Rev Microbiol 2013;11:227-38.

17. Rusconi F, Zugna D, Annesi-Maesano I, et al. Mode of delivery and asthma at school age in 9 European Birth Cohorts. Am J Epidemiol 2017;185:465-73.

18. Thavagnanam S, Fleming J, Bromley A, et al. A meta-analysis of the association between Caesarean section and childhood asthma. Clin Exp Allergy 2008;38: 629-33.

19. Stokholm J, Blaser MJ, Thorsen J, et al. Maturation of the gut microbiome and risk of asthma in childhood. Nat Commun 2018;9:141.

20. Abrahamsson TR, Jakobsson HE, Andersson AF, et al. Low gut microbiota diversity in early infancy precedes asthma at school age. Clin Exp Allergy 2014;44: 842-50.

21. Bisgaard H, Li N, Bonnelykke K, et al. Reduced diversity of the intestinal microbiota during infancy is associated with increased risk of allergic disease at school age. J Allergy Clin Immunol 2011;128:646-52.e1-5.

22. Fouhy F, Guinane CM, Hussey S, et al. High-throughput sequencing reveals the incomplete, short-term recovery of infant gut microbiota following parenteral antibiotic treatment with ampicillin and gentamicin. Antimicrob Agents Chemother 2012;56:5811-20.

23. Fanaro S, Chierici R, Guerrini P, et al. Intestinal microflora in early infancy: composition and development. Acta Paediatr Suppl 2003;91:48-55.

24. Kull I, Almqvist C, Lilja G, et al. Breast-feeding reduces the risk of asthma during the first 4 years of life. J Allergy Clin Immunol 2004;114:755-60.

25. Hanski I, von Hertzen L, Fyhrquist N, et al. Environmental biodiversity, human microbiota, and allergy are interrelated. Proc Natl Acad Sci U S A 2012;109:8334-9.

26. Stein MM, Hrusch CL, Gozdz J, et al. Innate immunity and asthma risk in Amish and Hutterite farm children. N Engl J Med 2016;375:411-21.

27. Ottman N, Ruokolainen L, Suomalainen A, et al. Soil exposure modifies the gut microbiota and supports immune tolerance in a mouse model. J Allergy Clin Immunol 2019;143(3):1198-206.e12.

28. Byrd AL, Belkaid Y, Segre JA. The human skin microbiome. Nat Rev Microbiol 2018;16:143-55.

29. Oh J, Byrd AL, Park M, et al. Temporal stability of the human skin microbiome. Cell 2016;165:854-66. 
30. Fyhrquist N, Salava A, Auvinen P, et al. Skin Biomes. Curr Allergy Asthma Rep 2016;16:40.

31. Belkaid Y, Tamoutounour S. The influence of skin microorganisms on cutaneous immunity. Nat Rev Immunol 2016;16:353-66.

32. Belkaid Y, Segre JA. Dialogue between skin microbiota and immunity. Science 2014;346:954-9.

33. Panther DJ, Jacob SE. The importance of acidification in atopic eczema: an underexplored avenue for treatment. J Clin Med 2015;4:970-8.

34. Sanchez Rodriguez R, Pauli ML, Neuhaus IM, et al. Memory regulatory T cells reside in human skin. J Clin Invest 2014;124:1027-36.

35. Scharschmidt TC, Vasquez KS, Truong HA, et al. A wave of regulatory t cells into neonatal skin mediates tolerance to commensal microbes. Immunity 2015;43: 1011-21.

36. Volz T, Skabytska Y, Guenova E, et al. Nonpathogenic bacteria alleviating atopic dermatitis inflammation induce IL-10-producing dendritic cells and regulatory $\operatorname{Tr} 1$ cells. J Invest Dermatol 2014;134:96-104.

37. Laborel-Preneron E, Bianchi P, Boralevi F, et al. Effects of the Staphylococcus aureus and Staphylococcus epidermidis secretomes isolated from the skin microbiota of atopic children on CD4+ T cell activation. PLoS One 2015;10: e0141067.

38. Fyhrquist N, Ruokolainen L, Suomalainen A, et al. Acinetobacter species in the skin microbiota protect against allergic sensitization and inflammation. J Allergy Clin Immunol 2014;134:1301-9.e11.

39. Weidinger S, Novak N. Atopic dermatitis. Lancet 2016;387:1109-22.

40. Apfelbacher CJ, Diepgen TL, Schmitt J. Determinants of eczema: populationbased cross-sectional study in Germany. Allergy 2011;66:206-13.

41. Akiyama M. FLG mutations in ichthyosis vulgaris and atopic eczema: spectrum of mutations and population genetics. Br J Dermatol 2010;162:472-7.

42. Nutten S. Atopic dermatitis: global epidemiology and risk factors. Ann Nutr Metab 2015;66(Suppl 1):8-16.

43. Kong HH, Oh J, Deming $\mathrm{C}$, et al. Temporal shifts in the skin microbiome associated with disease flares and treatment in children with atopic dermatitis. Genome Res 2012;22:850-9.

44. Leyden JJ, Marples RR, Kligman AM. Staphylococcus aureus in the lesions of atopic dermatitis. Br J Dermatol 1974;90:525-30.

45. Huang JT, Abrams M, Tlougan B, et al. Treatment of Staphylococcus aureus colonization in atopic dermatitis decreases disease severity. Pediatrics 2009;123: e808-14.

46. Savinko T, Lauerma A, Lehtimäki S, et al. Topical superantigen exposure induces epidermal accumulation of CD8+ T cells, a mixed Th1/Th2-type dermatitis and vigorous production of IgE antibodies in the murine model of atopic dermatitis. J Immunol 2005;175:8320-6.

47. Wickersham M, Wachtel S, Wong Fok Lung T, et al. Metabolic stress drives keratinocyte defenses against Staphylococcus aureus infection. Cell Rep 2017;18: 2742-51.

48. Byrd AL, Deming C, Cassidy SKB, et al. Staphylococcus aureus and Staphylococcus epidermidis strain diversity underlying pediatric atopic dermatitis. Sci Transl Med 2017;9 [pii:eaal4651].

49. Yeung M, Balma-Mena A, Shear N, et al. Identification of major clonal complexes and toxin producing strains among Staphylococcus aureus associated with atopic dermatitis. Microbes Infect 2011;13:189-97. 
50. Zeeuwen PL, Ederveen TH, van der Krieken DA, et al. Gram-positive anaerobe cocci are underrepresented in the microbiome of filaggrin-deficient human skin. J Allergy Clin Immunol 2017;139:1368-71.

51. Denner DR, Sangwan N, Becker JB, et al. Corticosteroid therapy and airflow obstruction influence the bronchial microbiome, which is distinct from that of bronchoalveolar lavage in asthmatic airways. J Allergy Clin Immunol 2016;137: 1398-405.e3.

52. Frei R, Lauener RP, Crameri R, et al. Microbiota and dietary interactions: an update to the hygiene hypothesis? Allergy 2012;67:451-61.

53. Herbst $T$, Sichelstiel A, Schär C, et al. Dysregulation of allergic airway inflammation in the absence of microbial colonization. Am J Respir Crit Care Med 2011; 184:198-205.

54. Marsland BJ, Trompette A, Gollwitzer ES. The gut-lung axis in respiratory disease. Ann Am Thorac Soc 2015;12(Suppl 2):S150-6.

55. Schuijs MJ, Willart MA, Vergote K, et al. Farm dust and endotoxin protect against allergy through A20 induction in lung epithelial cells. Science 2015;349:1106-10.

56. Gollwitzer ES, Saglani S, Trompette A, et al. Lung microbiota promotes tolerance to allergens in neonates via PD-L1. Nat Med 2014;20:642-7.

57. Huang YJ, Nelson CE, Brodie EL, et al. Airway microbiota and bronchial hyperresponsiveness in patients with suboptimally controlled asthma. J Allergy Clin Immunol 2011;127:372-81.e1-3.

58. Haahtela $\mathrm{T}$, Laatikainen $\mathrm{T}$, Alenius $\mathrm{H}$, et al. Hunt for the origin of allergy comparing the Finnish and Russian Karelia. Clin Exp Allergy 2015;45:891-901.

59. Ruokolainen L, Paalanen L, Karkman A, et al. Significant disparities in allergy prevalence and microbiota between the young people in Finnish and Russian Karelia. Clin Exp Allergy 2017;47:665-74.

60. Turnbaugh PJ, Ley RE, Mahowald MA, et al. An obesity-associated gut microbiome with increased capacity for energy harvest. Nature 2006;444:1027-31.

61. Roesch LF, Lorca GL, Casella G, et al. Culture-independent identification of gut bacteria correlated with the onset of diabetes in a rat model. ISME J 2009;3(5): 536-48.

62. Thorburn AN, McKenzie Cl, Shen S, et al. Evidence that asthma is a developmental origin disease influenced by maternal diet and bacterial metabolites. Nat Commun 2015;6:7320.

63. Trompette A, Gollwitzer ES, Yadava K, et al. Gut microbiota metabolism of dietary fiber influences allergic airway disease and hematopoiesis. Nat Med 2014;20(2): 159-66.

64. Lelouard H, Fallet M, de Bovis B, et al. Peyer's patch dendritic cells sample antigens by extending dendrites through M cell-specific transcellular pores. Gastroenterology 2012;142:592-601.e3. 